\title{
Simulation in Quality Management - An Approach to Improve Inspection Planning
}

\author{
H.-A. Crostack, M. Höfling, J. Liangsiri
}

Production is a multi-step process involving many different articles produced in different jobs by various machining stations. Quality inspection has to be integrated in the production sequence in order to ensure the conformance of the products. The interactions between manufacturing processes and inspections are very complex since three aspects (quality, cost, and time) should all be considered at the same time while determining the suitable inspection strategy. Therefore, a simulation approach was introduced to solve this problem.

The simulator called QUINTE [the QUINTE simulator has been developed at the University of Dortmund in the course of two research projects funded by the German Federal Ministry of Economics and Labour (BMWA: Bundesministerium für Wirtschaft und Arbeit), the Arbeitsgemeinschaft industrieller Forschungsvereinigungen (AiF), Cologne/Germany and the Forschungsgemeinschaft Qualität, Frankfurt a.M./Germany] was developed to simulate the machining as well as the inspection. It can be used to investigate and evaluate the inspection strategies in manufacturing processes. The investigation into the application of QUINTE simulator in industry was carried out at two pilot companies. The results show the validity of this simulator. An attempt to run QUINTE in a user-friendly environment, i.e., the commercial simulation software - Arena ${ }^{\circledR}$ is also described in this paper.

Keywords: simulation, quality, inspection strategies, manufacturing process.

NOTATION: QUINTE Qualität in der Teilefertigung (Quality in the manufacturing process)

\section{Introduction}

A major tool used to measure and assure quality is quality inspection. This inspection can assure that the products being produced meet the standards of quality and describe the quality levels. The objective of methodically planned inspection is to ensure regular quality inspection and its optimum integration into the production sequence.

Quality inspection can be a check made on each piece produced (100\% inspection) or a check made on a statistical sample of the lot. The inspection can be a mechanical or electronic measurement or a visual inspection. It can also be performed by the operator or worker making the part or component, by a second person who is responsible for measuring only, or performed entirely by computer-controlled measurement. These matters are combined into an inspection strategy. Each inspection strategy has its own pros and cons. Three important criteria are needed to evaluate the inspection strategies: quality, cost and time. The best inspection strategy should consider not only one criterion but all three of them.

However, to find this best integration of inspection into production is not an easy task. The cycle times, quality and manufacturing costs - depending on lot sizes or on work-in-process - are difficult to estimate and a process improvement is hard to identify without support. Therefore, simulation has become a powerful tool to solve this problem.

There have been some researches on simulation in the quality inspection area. Tannock [1] developed a simulation model in order to evaluate inspection strategies according to process capability and cost of quality (COQ). The quality costs and the Taguchi-based measure (Qmp) are then evaluated, according to the inspection strategy selected. He also used the simulation method [2] to prove the capability of providing an insight into the comparative patterns of cost associated with control charting for variables and alternative inspection strategies. The simulation results confirm that control charting of variables is much more efficient than 100 percent inspection at reducing losses caused by process trends or changes in the variability when known assignable causes are applied to the data.

Although many researches have been done on quality inspection, no exhaustive investigation of the inspection strategy in different planning factors with respect to quality, costs and time has been made. This report therefore presents the developed simulator named "QUINTE" [3, 4], which can be used to investigate and evaluate the inspection strategies with respect to quality, cost, and time in the manufacturing process. The paper also introduces the application of this simulator in industry and the integration to commercial simulation software. Before describing the simulator in detail, a section focusing on the fundamental concept of inspection strategies is presented.

\section{Inspection strategies}

The inspection strategy should be planned at the beginning of the product development phase together with process planning, in order to allow integration of the inspection planning into the quality planning. There are five main aspects that should be considered in inspection strategy planning [5]. The quality characteristics of the product that need to be inspected should also be defined and classified in advance.

\subsection{Inspection method}

The method used for inspection can be either an attribute method or a variable method. An attribute inspection checks whether the product is good or bad, while variable inspection obtains the quantitative value of the quality characteristic. To select the method used in the inspection, cost, time and 
application should be taken into account. While the attribute method is usually simple and inexpensive, the variable method gives adequate information for the purposes of process control.

\subsection{Inspection point}

The quality characteristics of products need to be inspected in order to prove the conformity of the parts with the demands. The earliest possible inspection point is located right after the production of the characteristic.

If an inspection is performed after every process, the scrap and rework costs are at a minimum because faulty items are identified before adding more costs to already defective material. However, it is more expensive to conduct the inspection in this way than to combine the inspections of many quality characteristics at a single inspection point. The reason for this is that the inspection time and cost, for example setup, queuing, and buffer, are high. If many characteristics are inspected together later in the process flow, the inspection time and cost will be lower. Then again, the scrap and rework costs will be higher. Therefore, if this intermediate inspection is done either too often or too late, unnecessary costs will occur.

The choice of the inspection point is based on a number of criteria such as inspection costs, damage risk, accessibility of the characteristic, increase in the product value, etc. Also the fact that some parts cannot be inspected when they are already assembled must be considered.

\subsection{Inspection extent}

A decision about the extent of the measurements must be carried out. This aspect of inspection strategy directly influences inspection and failure cost. It ranges from no test to random and intermittent tests, and all the way to a $100 \%$ test.

The $100 \%$ inspection is usually used for the final test of critical or complex products. It may also be used when the process capability is inherently too poor to meet product specification. To conduct $100 \%$ inspection is very costly and time consuming, even though the entire products are sorted.

Sample inspection is carried out according to externally valid standards or company internal regulations. When choosing the inspection extent, prior knowledge of the product is important, e. g., the importance of the feature for product quality, and process capability.

No inspection is used when there is already adequate evidence that the product conforms, and hence no further inspection is needed. While no inspection or sample inspection gives benefits in inspection cost and time, the company should bear in mind that it includes the risk of declaring the lot good even if it might contain defects in the lot.

\subsection{Inspection location and personnel}

Inspection can be performed either at the machine or at a special inspection location. In some cases, the operator may be the only person who should make the inspection. In other cases, the product might pass through an inspection or test station, where inspectors or testers make further inspections. Or such inspections might be made by automatic quality-control equipment and the data is automatically processed and used for adjustment of the process.
If the inspection is performed at the machine, the advantages are that no transportation is necessary and the feedback on error can be done quickly. However, the worker might spend more time on inspection and less time on production. Therefore, machine utilisation can decrease and the cycle time and manufacturing cost can increase. The accuracy of inspection might be low since the worker has to both operate the machine and inspect the parts. Moreover, it is not economical to have testing equipment at every production process or line, if the testing equipment is expensive.

The other hand, if the inspection is conducted at a special inspection location, the accuracy and capability of the inspection process are higher, as the inspection environment can be controlled. However, the cons of this alternative are higher transportation costs and higher cycle time, since the parts have to be transported and wait for inspection.

\subsection{Inspection equipment}

The capability of the inspection device (measurement accuracy) and the tolerance of the inspected characteristics are the main selection criteria. Furthermore, acquisition cost, capacity and other elements are taken into account. Most of the time, high capability devices are expensive and difficult to handle.

As mentioned above, the inspection strategies have drastic effects on the performance of production processes in terms of production cost, cycle time, and product quality. These impacts vary from one inspection strategy to another. Therefore, choosing a good inspection strategy can be a complicated decision. The inspection of a single process can influence the other processes in the production in such a way that would be hardly possible to predict the effects of different inspection strategies analytically. Thus, simulation can be a powerful tool to evaluate various inspection strategies.

\section{The "QUINTE" simulator}

Most general-purpose simulators focus on production and material handling systems. However, they are not directly aimed at the quality area, so some features which are valuable to quality engineers are lacking. Therefore, a simulator called QUINTE has been developed at the University of Dortmund and RIF e.V., Germany. The simulator focuses on the inspection strategy and its interaction with the production process. It is designed to investigate the impact of different inspection strategies on manufacturing cost, cycle time, and product quality. The flow chart of QUINTE components is shown in Fig. 1.

Initially, the model of the machining process is characterized by its own statistical distribution, for example, by a normal distribution with a certain standard deviation $\sigma$ and mean $\mu$, depending on the current process capability. Then, according to the statistical model, QUINTE randomly simulates the quality characteristic value of the given process.

QUINTE models the distributions dynamically since the process capability is not constant over time. The expected value can glide from its original value or the deviation can increase because of failure, wear of the tool, etc. This changed distribution can be restored or improved by setting up and 


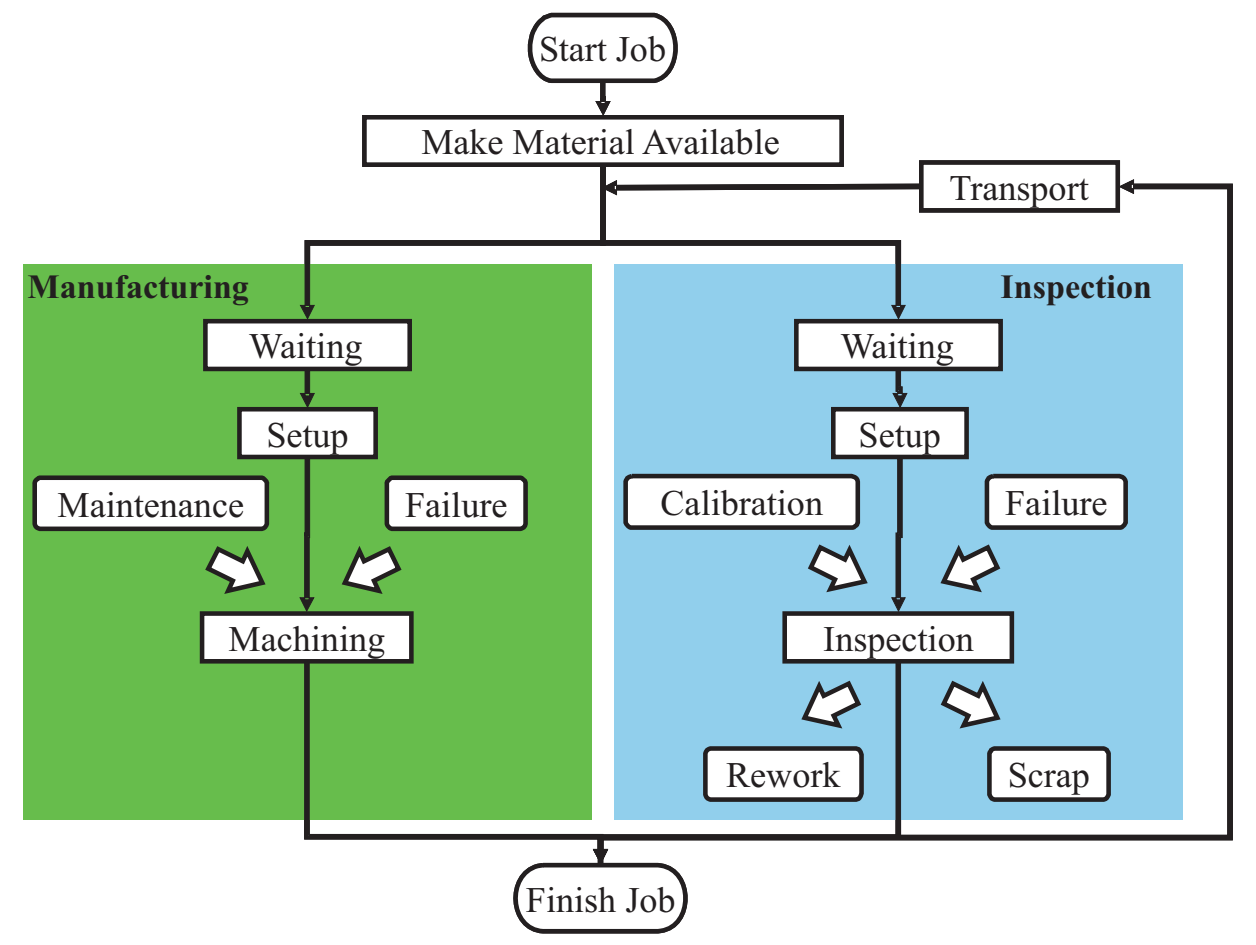

Fig. 1: Components of QUINTE

maintenance. The disturbance of machining process is modelled in two ways: failure and maintenance.

The obtained characteristic value denotes the actual value of a characteristic of a manufactured part. Once this characteristic value is acquired, it is stored in the database and is used for the inspection simulation.

The inspection process is simulated in a similar way as the manufacturing process. Due to bias and precision, the value given by the inspection tool may differ from the true value. The capability of the inspection process is described by a statistical distribution, for example, a normal distribution. A standard deviation $\sigma_{\text {insp }}$ and a mean $\mu_{\text {insp }}$ are assigned for each inspection process. The mean $\mu_{\text {insp }}$ is not a fixed value because the process is used to find out what value is produced.
Thus, the machined characteristic value is used as a mean of the inspection process. The capability of the inspection process is changed according to time in the same way as in the manufacturing process. Therefore, the expected value can glide from its original value or the deviation can increase. The distribution can be restored or improved by setting up and calibration. Fig. 2 shows a clear example of the inspection of characteristic value $x_{i}$. QUINTE randomly generates the inspected value from the specified distribution. The inspected value will be compared with the specification limit, thus the decision is made whether or not the part conforms.

Furthermore, the occurrences of decision errors (type I and type II error) can also be simulated by QUINTE. Because of the variation in inspection processes, there is a possibility

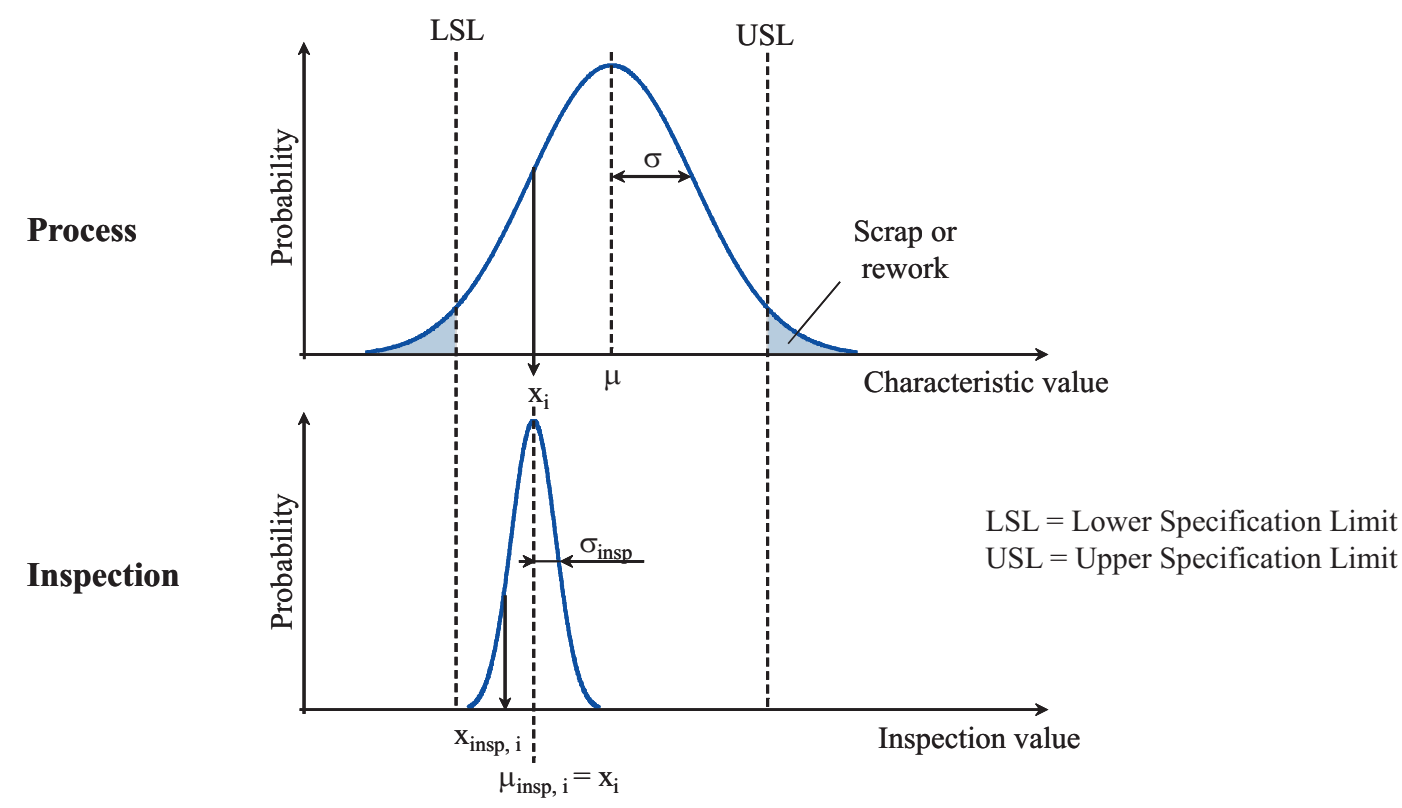

Fig. 2: Inspection of characteristic value $x_{i}$ 
that a wrong decision can be made. A type I error is an error when a good part is wrongly declared as a bad part. On the other hand, a type II error is an error when a bad part is declared as a good part.

After the decision is made, the part that is declared as a conformed part continues on its production sequence. Scraps must be sorted out and a new job must be started to replace the scraps if needed. Rework parts can be handled in two ways. The rework parts can be sent back to the preceding process or processes and the operation can be repeated. Another option is to repair the part in a separate rework area. At the end of the simulation runs, the simulation output is used in the evaluation of the inspection strategies.

\section{Industrial application}

The application of QUINTE in industry was tested in collaboration with two pilot companies [6]. The results from the simulator are presented in this section. However, due to the companies' confidentiality policies, the names of the companies are not presented and the results presented here are not concrete values but only relative values.

\subsection{Example 1}

This pilot company is a manufacturer of mobile hydraulic products. Nine different products from 19 manufacturing stations, 8 inspection stations, and 1 manufacturing/inspection tool store were chosen for the simulation experiments. Each product had different production sequences and different quality characteristics. In the simulation modelling, 268 inspection tools were considered. Six sampling inspections were defined in the modelling. Five of them were based on DIN EN ISO 2859 and one sampling inspection was done every $50^{\text {th }}$ part. The transportation time between stations was assumed to be 100 seconds.

The combinations of these inspection aspects were combined into inspection strategies, and ten of these strategies were chosen for the simulation experiments. The results of the simulation experiments are shown in Figs. 3 and 4 . However, these results cannot be compared with the current situation at the company, since the company does not implement any solid inspection strategy. The inspection is done from operators' experience.

The results show that there is no significant difference between all strategies in terms of manufacturing cost and rework
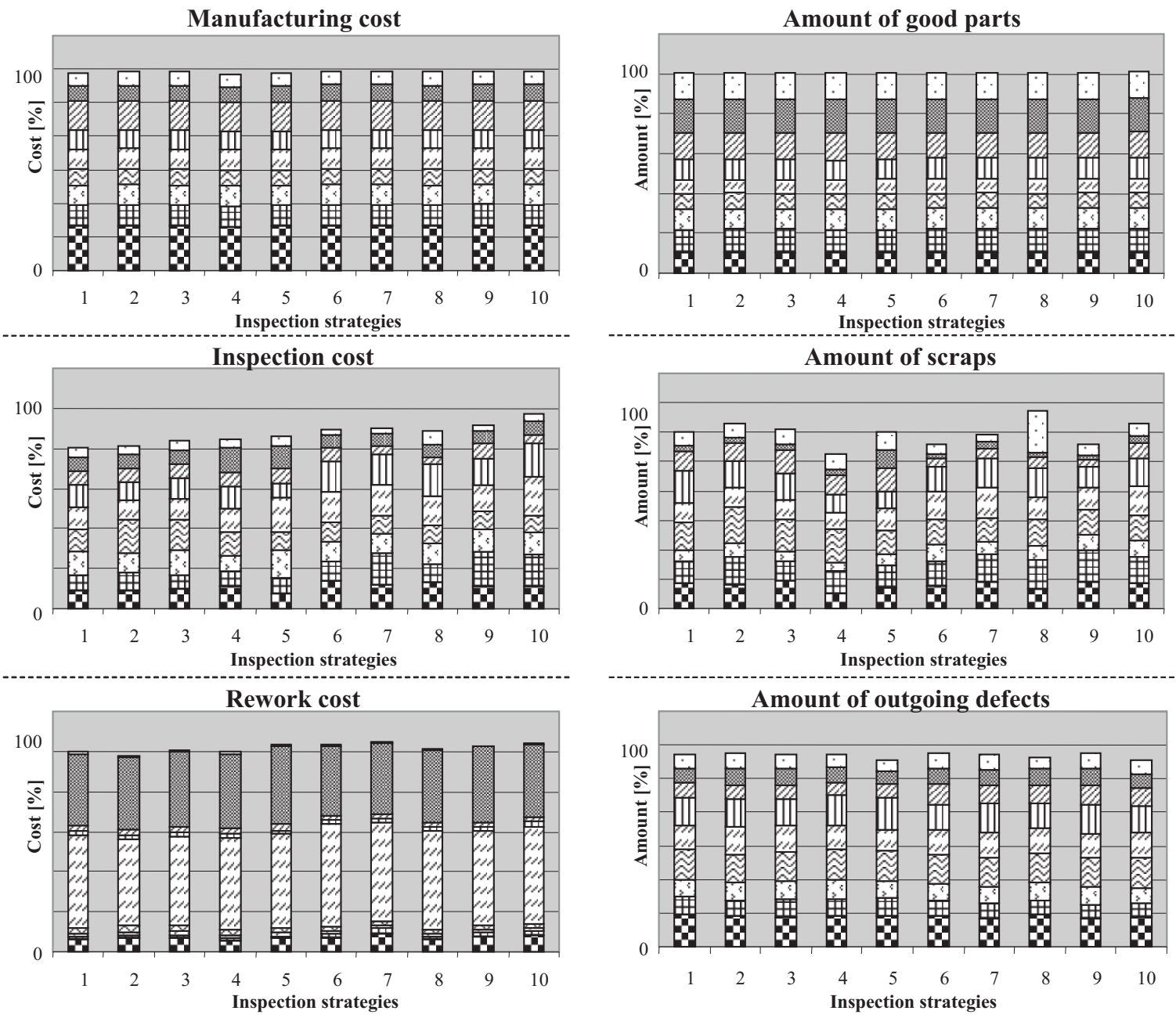

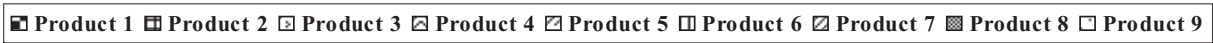

Fig. 3: Simulation results of Company 1 (1) 
cost. However, clear differences appear in quality (amount of scrap) and cycle time. The best inspection strategy is alternative no. 4 , since it gives the best quality and cycle time. From this result, the company can realize where the potential improvements are.

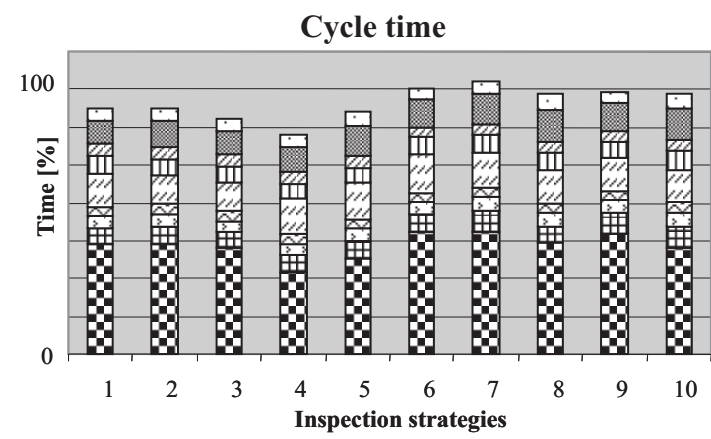

Product 1 田Product 2 Product 3 园 Product 4

$\square$ Product 5 m Product $6 \square$ Product 7 : Product 8 $\square$ Product 9

Fig. 4: Simulation results of Company 1 (2)

\subsection{Example 2}

The manufacture of stub shafts in the second company was chosen as an area of study. Three types of products, Products 10,19 , and 20, were taken into consideration. The chosen part of the factory consisted of six manufacturing stations, one inspection room, and two manufacturing/ inspection tool stores. The transportation time between manufacturing stations was assumed to be 20 seconds, while the transportation time between a manufacturing station and an inspection station or the tool store was 300 seconds.

In the simulation modelling, 155 inspection tools were considered. They were located at one of the following places: the manufacturing station, the inspection room, or the tool store. Six sampling inspections were defined in the modelling. Four of them were based on DIN EN ISO 2859, and two sampling inspections were done every $50^{\text {th }}$ and $150^{\text {th }}$ part, respectively. With the same procedure as for example 1 , ten inspection strategies were selected for the simulation experiments plus the other three inspection strategies, namely: 1) the current strategy of the company, 2) no inspection at all, and 3) $100 \%$ inspection after each process.

The result for cycle time is shown in Fig. 5. It is obvious that no inspection alternative (No. 11) gives the lowest cycle time, while $100 \%$ inspection gives the highest cycle time. The cycle time for the $100 \%$ inspection alternative (No. 13) is not presented in the figure due to the extreme bar height in the diagram.

As long as costs are concerned, there are no apparent differences for manufacturing cost and inspection cost. However, the current strategy gives a higher rework cost and a higher amount of scrap than the ten other selected strategies. The reason is that the three characteristics were $100 \%$ inspected and the accuracy of the chosen inspection tool was not adequate in the current strategy, while the sampling inspections with the same inspection tool were done in other alternatives. For this reason, there are more inspection errors

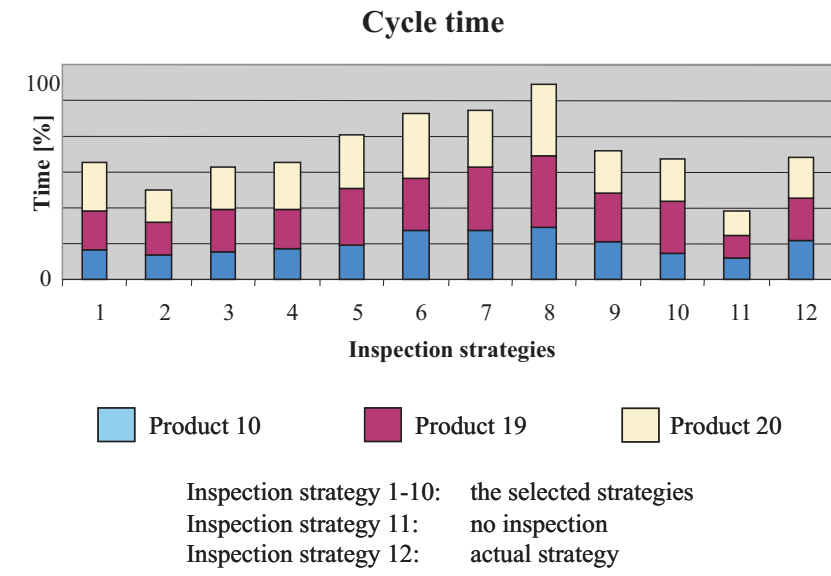

Fig. 5: Simulation results of Company 2

in the current strategy than others. Aside from this, the currently used strategy at this company is still among the best strategies in terms of overall performance. The current strategy can be improved by replacing the bad parameters with good parameters from other analysed strategies, e.g. the inspections of some characteristics can be changed to sampling inspection.

\section{QUINTE in the new environment}

The QUINTE simulator was originally built on $\mathrm{C} / \mathrm{C}++$ language and it was successfully tested in industry. However, there are some minor drawbacks in QUINTE; for example, there is no animation for the simulation, only limited process distributions can be modelled, and it is difficult to modify or enhance other components or functions in QUINTE without strong knowledge of $\mathrm{C} / \mathrm{C}++$. In order to overcome these shortcomings, QUINTE was transferred into a commercial simulation software called Arena ${ }^{\circledR}$. With this new QUINTE environment, the user can create and animate the processes, use Arena's statistical analyzer, and use other user-friendly Arena functions. Moreover, QUINTE can easily be modified or enhanced by the developer without a need for programming, since Arena is very easy to use with its point and click interface and fill in the blank dialog boxes.

Most of QUINTE's functions are now placed in Arena's template, as shown in Figure 6. The QUINTE template consists of 9 modules. The first module is the "General Process" module. This module is designed for any processes in production, such as the manufacturing and inspection process. The user can assign the process name, processing time, cost allocation, information about failure and maintenance. There are two modules for the quality characteristic assignment: "Attribute Characteristic" and "Variable Characteristic" modules. The information about a characteristic, its distribution or conformity rate, and the specifications can be defined here. As described earlier, QUINTE can model these distributions dynamically. The deviation of the distribution can be assigned under the "Distribution Change..." dialogue box (Fig. 6).

The information about inspection equipment can be added in the "Inspection - Attribute" or "Inspection - Variable" module, according to the type of quality characteristic that the equipment can measure. In these modules, the accuracy 


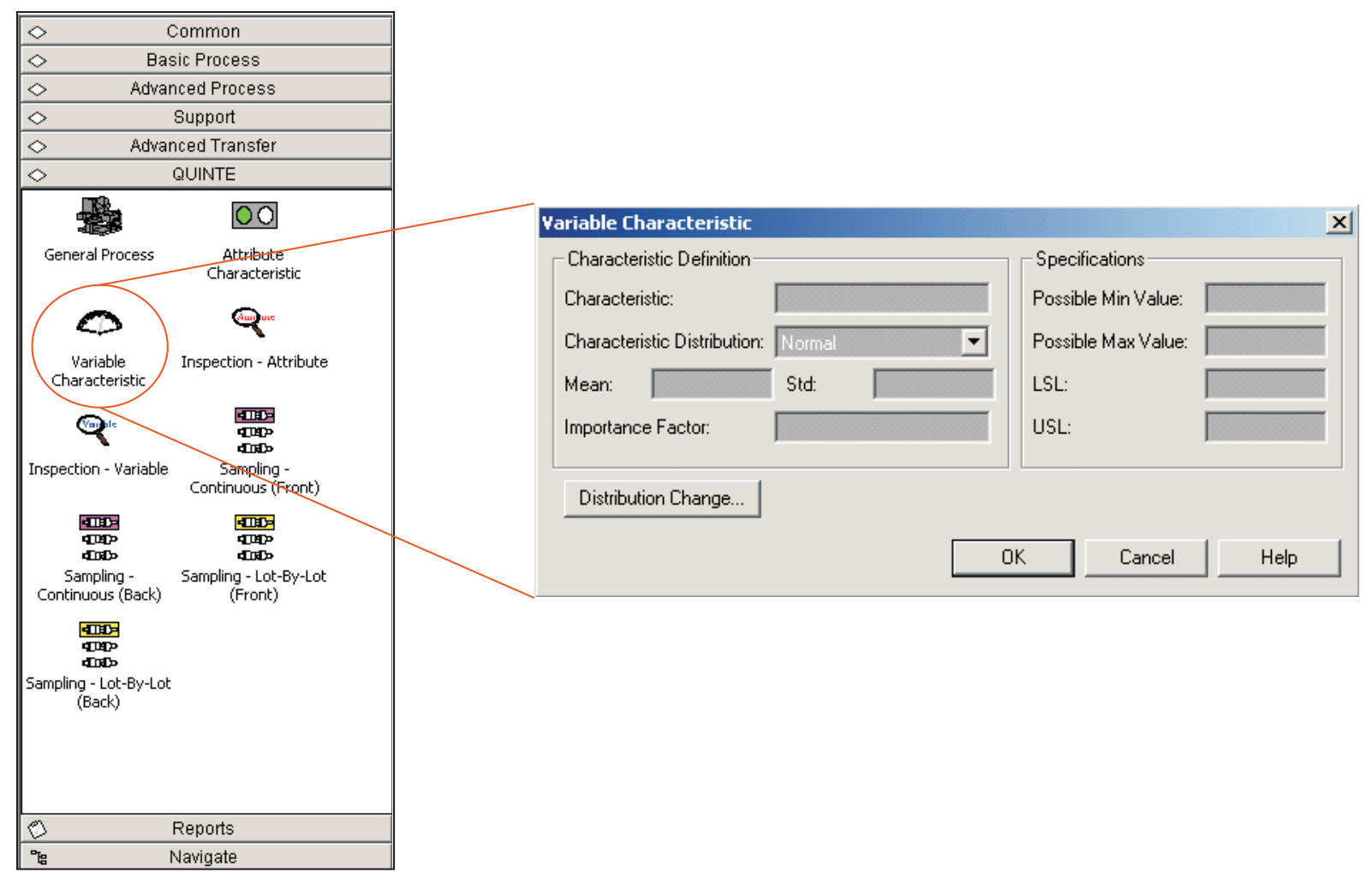

Fig. 6: QUINTE template in Arena ${ }^{\circledR}$

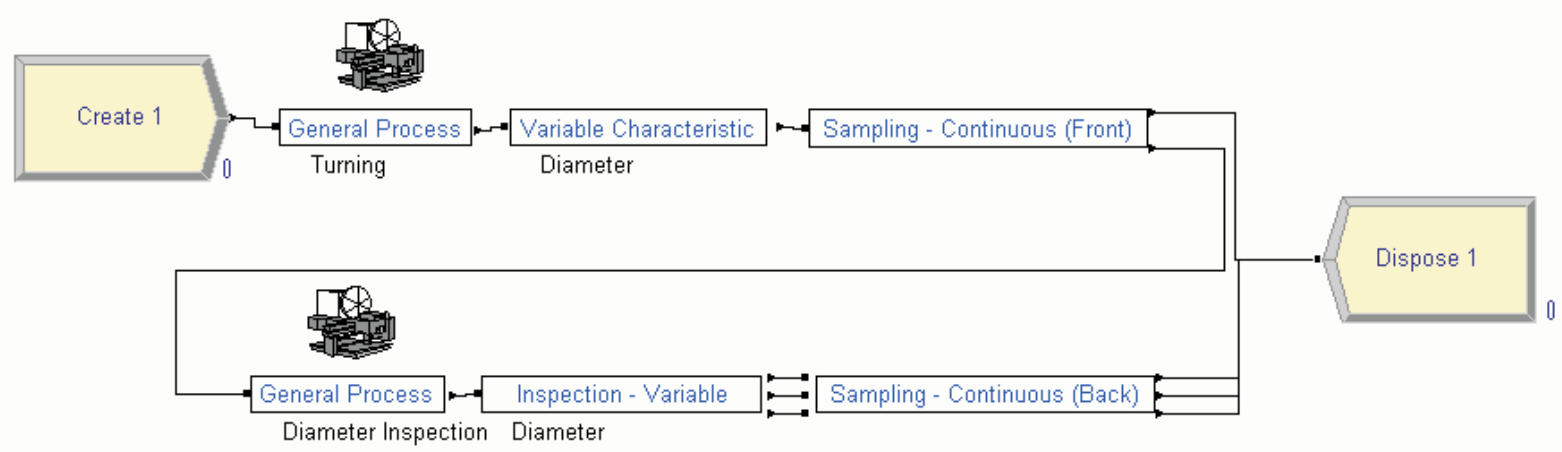

Fig. 7: Modelling example

of the inspection equipment must be defined. The user also has the possibility to add the change in inspection accuracy over time.

The last four "Sampling" modules are used to insert the sampling inspection into the model. The two options for sampling inspection are continuous and lot-by-lot. The continuous sampling inspection module is based on Dodge's AOQL plan for continuous production (CSP-1) [7]. The application of this plan aims for continuous production, since the formation of inspection lots for lot-by-lot acceptance is impractical and costly. The lot-by-lot sampling inspection module was built according to a single sampling scheme.

The simulation model can be easily built with this QUINTE template and other Arena templates. The user can build the model by dragging and dropping the required modules into the modelling space, can fill in the necessary information, and connect the modules together in the same order as the process flow. A simple example of how to model in Arena is illustrated in Fig. 7. The example shows the manufacture and inspection of a shaft. Shafts enter the simulation model by the "Create" module and pass through a turning machine, which produces the diameter as a quality characteristic. After the characteristic is produced, the shafts are sampled. Those which are not to be inspected go to the next production step, which in this example is the disposal step. Those which must be inspected are sent to the diameter inspection process and then go to the next step.

The usability of QUINTE template has been preliminarily tested with some examples and the results show the validity of this new version of QUINTE.

\section{Conclusion}

The QUINTE simulator, which simulates the manufacturing process and inspection was successfully developed and validated. The application in two pilot companies proves 
that this simulator can be used to investigate and evaluate different inspection strategies. The result from QUINTE enables the company to choose the most suitable inspection strategy according to the company's goal. The simulator can also support the management in justifying of investment in inspection equipment or in a manufacturing process, for example, by illustrating the consequences of changes in the uncertainty of inspection equipment. Furthermore, the simulator was reconstructed in Arena simulation software. This new environment makes it easier for users to build the model and get additional advantages from Arena's functions.

Although QUINTE is a beneficial simulator, some potential improvements can still be made. This tool can be applied extensively in other areas, such as assembly processes and commissioning processes although the main concentration of QUINTE is currently only on manufacturing processes.

\section{References}

[1] Tannock, J. D. T.: "Choice of Inspection Strategy Using Quality Simulation." International Journal of Quality \& Reliability Management, Vol. 12 (1995), No. 5, p. 75-84.

[2] Tannock, J. D. T.: "An Economic Comparison of Inspection and Control Charting Using Simulation." International Journal of Quality \& Reliability Management, Vol. 14 (1997), No. 7, p. 687-699.

[3] Crostack, H.-A., Heinz, K., Nürnberg, M., Nusswald, M.: "Evaluating Inspection Strategies by Simulation." Manufacturing Systems, Vol. 29 (1999), No. 5, p. 421-425.

[4] Crostack, H.-A., Mayer, M., Höfling, M.: "Optimization of Inspection and Test Planning - Report on a German Research Project." $17^{\text {th }}$ International Conference on Production Research, No. 67, Blacksburg, Virginia, USA, 2003.

[5] Pfeifer, T.: Production Metrology. Oldenbourg Verlag München Wien, 2002.

[6] Crostack, H.-A., Hermes, A., Höfling, M., Zielke, R., Heinz, K., Grünz, L., Mayer, M.: "QUINTE+ Optimierung der Prüfplanung nach Kosten und Durchlaufzeit mit Hilfe der Simulation. FQS-DGQ-Band Nr. 84-04. Frankfurt: FQS - Forschungsgemeinschaft Qualität e.V., 2004.

[7] Dodge, H. F.: "A Sampling Inspection Plan for Continuous Production." The Annals of Mathematical Statistics, Vol. 14, September 1947, p. 264-279.

Prof. Dr.-Ing. H.-A. Crostack

phone: +492319700101

fax: +492319700460

email: sekretariat@rif.fuedo.de

Dipl.-Ing. M. Höfling

phone: +492319700126

fax: +492319700460

email: mhoef@rif.fuedo.de

J. Liangsiri, M.Sc.

phone: +492319700107

fax: +492319700460

email: jliangsi@rif.fuedo.de

Dortmunder Initiative zur Rechnerintegrierten

Fertigung e.V. (RIF)

Joseph-von-Fraunhoferstr. 20

44227 Dortmund, Germany 